\title{
Optimal Segmentation of Brain Tumors using DRLSE Levelset
}

\author{
Usha Rani.N \\ Associate Professor \\ Vignan University \\ Vadlamudi(post) \\ Chebrolu(mdl) \\ Guntur(dist) \\ PIN-522213
}

\author{
Dr.P.V.Subbaiah \\ Professor \& Principal \\ Amritha Sai Institute \\ Of Technology \\ Kanchikacharla(post) \\ Krishna(Dist) \\ Andhra Pradesh
}

\author{
Dr.D.Venkata \\ Rao \\ Professor \& Principal \\ PPD College of \\ Engg\& Technology, \\ Surampalli, \\ Vijayawada-521 212 \\ Andhra Pradesh
}

\author{
Nalini.K \\ Assistant Professor \\ Vignan University \\ Vadlamudi(post) \\ Chebrolu(mdl) \\ Guntur(dist) \\ PIN-522213
}

\begin{abstract}
Image segmentation plays a vital role in medical image processing and computer vision. In case of medical scan images geometric level set functions perform accurate segmentation in good no of cases but develops irregularities during concave region evolution. These irregularities cause numerical errors and eventually destroy the stability of the evolution. In this paper, a new variational formulation known as distance regularization has a unique forward-and-backward (FAB) diffusion effect is used for the analysis of medical brain image scans which perform accurate segmentation in case of concavities. This method also eliminates the need of the costly re-initialization procedure. This method shows reliable and good convergence to the object boundaries with speed in case of concavities.
\end{abstract}

\section{General Terms}

Image processing, Image Segmentation Algorithms et. al.

\section{Keywords}

Segmentation, Medical image processing, Variational Levelset, Distance Regularized Level Set, Re-initialization, Convergence.

\section{INTRODUCTION}

Medical image segmentation is the process of labeling each pixel in a medical scan image data set to indicate its tissue type and provide information about the anatomical structure. The various problems in medical image segmentation are poorly defined boundaries, blur or weak edges, intensity inhomogeneity and inconsistency in image quality while scanning and variable object shapes. Hence the conventional static segmentation methods like thresholding, edge and region based methods are not used for the segmentation of medical images. In medical imaging dynamic model based active contour methods from the work of Terzopouli's.et.al [1] are suitable for image segmentation and further analysis of dynamic image data. Active contour models (or snakes), was first introduced by Kass et al. [2]-[3], and developed quickly in many directions. The contour based deformable methods are classified as parametric and geometric methods [4]. Geometric implicit levelsets are introduced by osher and Sethian. Active contours are curves defined within an image domain that can move under the influence of internal forces within the curve itself and external forces derived from the image data[5-7]. The internal and external forces are defined so that the curve will conform to an object boundary or other desired features within an image. Internal forces consider smoothness and leakage during curve evolution. External forces move the curve towards the object boundaries.

Parametric segmentation[8] or Snakes deals with potential force and various external forces like Gradient Vector Force or balloon forces for the curve evolution. In parametric method the parameters are to be specified explicitly whenever changes in shape occur during evolution. Though parametric methods are fast and userinteracive, specifying parameters is a difficult task. Whereas in case of implicit or geometric methods shape changes are handled automatically. In geometric or levelset methods the solution for image segmentation is curve evolution of active contours, starting with an initial curve in the 2-D and evolving it to the object boundaries i.e. steady state. This work aims at application of variational DRLSE method for segmenting tumors from the medical brain CT, MRI and PET image scans. Performance parameters like Convergence accuracy towards boundaries and computational speed of this method is compared to the enengy based variational method.

Given an image our objective is to detect the shapes of the objects in it.. An image I can be defined as a bounded real positive function on some (rectangular) domain $\mathbf{\Omega} \in \mathrm{R}^{2}$. Let object boundary is denoted by $\partial \Omega$. Let image I $€\{0,1,2, \ldots \ldots, 255\}$ referred to as a grayscale image. We denote the curve c: $[0,1]$ parameterized by arc length. We segment the defined image I into 2 regions. Then definition of segmentation is as follows. Let I can be an image, suppose $\Omega$ can be parameterized into $\Omega 1$ and $\Omega 2$ based on some image property $p$ like image intensity, texture and pattern curvature etc. Then I/ $\Omega 1$ differ from $\mathrm{I} / \Omega 2$. Segmentation objective is to find a curve $C € \Omega$ such that it partitions $\Omega=\Omega 1 \cup \underline{U} \Omega$. The validation of segmentation solution is model dependent and subjective [ 8-9].

The organization of this paper is as follows. Section II presents the review of various geometric deformable models. Section III discusses the DRLSE variational method. Experimental results compare the performance of DRLSE and Energy based variational methods in terms of computation time, and accuracy in the convergence towards object boundaries. 


\section{GEOMETRIC DEFORMABLE MODELS}

Geometric or implicit active contour method is a model based one uses the curve evolution technique. Curve always evolves in the normal direction. Geometrical snakes, on the other hand, are represented implicitly as the zero-level sets of higherdimensional surfaces, and the updation is performed on the surface function within the entire image domain.

\subsection{Levelset Method}

Geometric active contour methods are based on the curve evolution and the levelset methods. In this method levelset function is represented as $1 \mathrm{D}$ higher, and basic contour which is represented around the object to be segmented is represented as the zero levelset of an implicit levelset function. The basic 2D curve around the object and 3D levelset function representation is shown Fig 1(a) and (b) respectively.

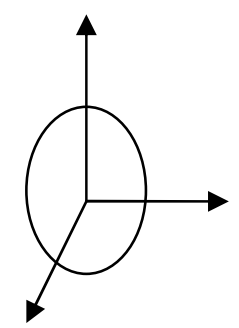

(a)

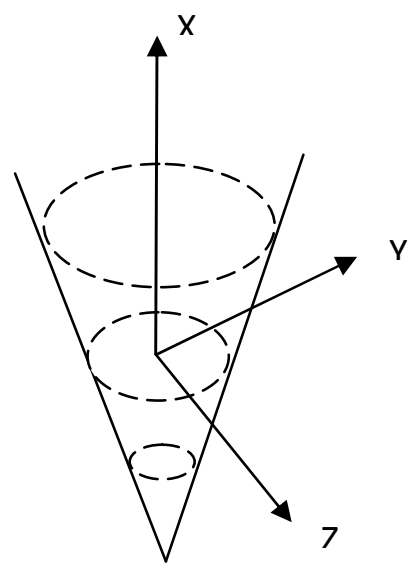

(b)

\section{Fig 1: Contour and Levelset Representation}

\section{(a) 2D Contour around the Object}

(b) 3D Higher order Levelset function

Fig.1(a) is the contour around the object evolves depending on the external and internal forces. Fig.1(b) represents the levelset function which incorporates this curve as zero levelset and evolves in 3-D. This levelset function is to be evolved using heat equation known as levelset PDE as follows.

The levelset pde is given by the equation (1) Which includes both spatial and temporal derivatives.

$$
\frac{\partial \phi}{\partial t}+F|\nabla t|=0
$$

Where $\mathrm{F}$ is the speed function, $\nabla$ is the gradient operator

The above pde is implemented in discrete form[ 6] given in eqn(2).The spatial resolution in $\mathrm{x}$ - and $\mathrm{y}$ - direction is $\Delta x$ and $\Delta y$ is represented with grid nodes $\mathrm{i} j$.The general notation of levelset function is ${\delta^{\mathrm{i}}}_{\mathrm{ij}}$ is approximation to the solution ( $\phi i h, j h, n \Delta t$ ), where $\Delta t$ is the time step.

$$
\frac{\Phi_{i, j}^{n+1}-\Phi_{i, j}^{n}}{\Delta t}+F\left(\nabla_{i, j} \Phi_{i, j}^{n}\right)=0
$$

This representation can 1) break or merge during evolution naturally and 2) it remains a function on a fixed grid hence numerical methods can be applied efficiently. The advantage of (Eulerian) this formulation is $\phi(x, t)$ remain a function as it evolves hence it can be represented on a discrete grid. The spatial and temporal derivatives in a pde (1) can be mathematically represented using difference approximations. Central differences are used for Spatial derivatives $\partial \phi / \partial x, \partial \phi / \partial y$ and forward difference is used for temporal derivatives $\partial \phi / \partial t$ is used. Although level set methods have been used to solve a wide range of medical imaging problems, their applications have been suffered with the irregularities of the LSF that are developed during the level set evolution. The pde can develop shocks during sharp and flat shape evolution which needs re-initialization. Reinitialization is performed by periodically stopping the evolution and reshaping the degraded LSF as a signed distance function [5-7]. Reinitialization raises serious problems and also affects the numerical accuracy in an undesirable way. Hence the Eulerian PDE is converted as Variational level set method based on energy minimization doesn't need reinitialization and are convenient for adding external shape, color or texture information into the model.

\subsection{Variational Method}

The variational formulation for geometric active contours forces the level set function to be close to a signed distance function, and therefore completely eliminates the need of the costly reinitialization procedure. Our variational formulation consists of an internal energy term that penalizes the deviation of the level set function from a signed distance function, and an external energy term that drives the motion of the zero level set toward the desired image features, such as object boundaries. The resulting evolution of the level set function is the gradient flow that minimizes the overall energy function. The advantage of this method over conventional levelsets is it is fast since larger time steps can be used in evolution pde. The mathematical formulation of variational method is represented as follows.Let $\mathrm{R}$ be the image domain, $\Omega$ be the subset of index R. $\partial \Omega$ is the boundary.

The initial levelset function $\emptyset$ is defined as

$$
\begin{aligned}
\Phi_{0}(x, y) & =-c_{o}(x, y) \in(\Omega-\partial \Omega) \\
& =0 \quad(x, y) \in(\partial \Omega) \\
& =c_{o}(x, y) \in(\mathfrak{R}-\Omega)
\end{aligned}
$$


In variational formulation $[12,13] \mathrm{P}(\phi)$ penalty term (internal energy) function of $\varphi$ penalizes the deviation of the LSF from a signed distance function. The penalty term not only eliminates the need for reinitialization, but also allows the use of a simpler and more efficient numerical scheme in the implementation.

The penalty term is defined as follows

$$
\mathrm{P}(\phi)=\int_{\Omega} \frac{1}{2}(|\nabla \Phi|-1)^{2} d x d y
$$

The energy function is defined as follows

$$
\mathrm{E}(\phi)=\mu \mathrm{P}(\Phi)+\mathrm{E}_{m}(\Phi)
$$

Where $\mu>0$ is a constant, controls the deviation of $\phi$ from SDF. $\mathrm{E}_{m}(\Phi)$ is a certain energy that would drive the motion of the zero level curve of $\varphi$, hence called as external enengy depends on the image data. The external energy term in terms image parameters is defined as follows.

$$
\mathrm{E}_{g, \lambda, \vartheta}(\Phi)=\lambda L_{g}(\Phi)+v \mathrm{~A}_{g}(\Phi)
$$

Where $\mathrm{g}$ is a edge indicator function obtained from image data. where $\lambda>0$ and $v$ are constants, and the terms $A_{g}(\varphi)$ is introduced to speed up curve evolution. $L_{g}(\varphi)$ computes the length of the zero level curve of $\varphi$.In image segmentation, active contours are dynamic curves that moves toward the object boundaries. To achieve this goal, we explicitly define an external energy that can move the zero level curve toward the object boundaries. Let $I$ be an image, and $g$ be the edge indicator function defined by

$$
g=1 / 1+\left|\nabla G_{\sigma} * I\right|^{2}
$$

The total energy function is defined as follows

$$
\mathrm{E}(\phi)=\mu \mathrm{P}(\Phi)+\mathrm{E}_{g, \lambda, \vartheta}(\Phi)
$$

The Gateaux derivative [14](or first variation)] of the functional $E$, and the following evolution equation is in the variational levelsets which indicates the curve evolution w.r.t time.

$$
\frac{\partial \Phi}{\partial t}=-\frac{\partial \mathrm{E}}{\partial \Phi}-------(8)
$$

The penalizing term used as a metric to characterize how close a function $\varphi$ to a signed distance function in $\Omega € \mathrm{R}^{2}$ This metric will play a key role in our energy based variational level set formulation. However, this penalty term may not follow the SDF hence cause an undesirable side effect on the LSF when concavities involved This problem can be addressed in the distance regularized LSF.

\section{DISTANCE REGULARIZED LEVELSET EVOLUTION (DRLSE)}

The penalizing term in the previous method affects the numerical accuracy at concavities can be corrected by using regularizing term, The distance regularization term is defined with a potential function such that the derived level set evolution has a unique forward-and-backward (FAB) diffusion effect, which is able to maintain a desired shape of the level set function, particularly a signed distance profile near the zero level set. This yields a new type of level set evolution called distance regularized level set evolution (DRLSE).The level set evolution in $t$ is derived as the gradient flow that minimizes an energy functional with a distance regularization term and an external energy that drives the motion of the zero level set toward desired locations. The distance regularization effect eliminates the need for reinitialization and thereby avoids its induced numerical errors. Relatively large time steps can be used in the finite difference scheme to reduce the number of iterations, while ensuring sufficient numerical accuracy. This section presents the mathematical modeling of DRLSE.

The energy function $\mathrm{E}(\phi)$ in DRLSE as follows

$$
\mathrm{E}(\Phi)=\mu R_{p}(\phi)+\mathrm{E}_{\text {ext }}(\Phi)
$$

where $R_{p}(\phi)$ is the level set regularization term defined as follows, $\mu$ is a constant, and $\mathrm{E}_{\text {ext }}(\Phi)$ is the external energy that depends upon the data of interest (e.g., an image for image segmentation applications). The level set regularization term

$$
\begin{aligned}
& R_{p}(\phi) \text { is defined by } \\
& \quad R_{p}(\Phi) \cong \int_{\Omega} P(\nabla \Phi) d x \text {-.-------(10) }
\end{aligned}
$$

Where $\mathrm{P}$ is a potential energy function is designed such that it achieves a minimum when the zero level set of the LSF is located at desired position. 
The Gateaux derivative represents the evolution is defined as follows

$$
\frac{\partial \mathrm{E}(\Phi)}{\partial \Phi}=\mu \frac{\partial R_{p}(\Phi)}{\partial \phi}+\frac{\partial \mathrm{E}_{e x t}}{\partial \phi}
$$

$\mathrm{P}$ is a potential (or energy density) function $\mathrm{p} \in[0, \alpha], \mathrm{E}_{\mathrm{w}}(\phi)$ , external energy is designed such that it achieves a minimum when zero Level set of LSF $\phi$ is located at desired positions. Regularization term maintains the required $|\nabla \phi|=1$ in the vicinity of zero level set and also emerges smooth movement of the curve. The double well potential function with $|\nabla \phi|=0$ is responsible for the forward and backward (FAB) diffusions in case of conventional levelset. The DRLSE not only eliminates the need for reinitialization, but also allows the use of more general functions as the initial Level Set Functions. This method is more efficient in segmenting objects.

\section{RESULTS}

In this paper work is carried out by the application of levelsets to medical images. Particularly variational levelsets with enengy penalizing and distance regularization are used for the segmentation of brain tumors from CT,PET and MRI scan images. Segmentation of brain tumors using variational method with enengy penalizing and distance regularization is shown in Figs 2,3\& 4 respectively. The quantitative analysis is represented in tables 1 and 2 respectively. The performance is compared in terms of computation time and the convergence of the model towards object boundaries when the curve evolves.

Fig.2. represents the qualitative analysis of segmenting tumors in both the methods. Column 1 indicates the original images with tumors represented in polygons or ellipse. Column 2 represents segmentation of tumors shown with red curve using DRLSE method. Column3 indicates the segmented tumors with variational method. In case of simple tumors both the methods perform well in segmenting tumors. The computation times of same images in both the methods is shown in table.1.DRLSE method uses less time as shown in table1 column2, compared to the variational method. Fig.3. represents the curve and levelset function evolution in case of DRLSE.Fig.3.(a) represents the initial contour incase of MRI with 2 tumors and (b) represents the curve evolution and segmentation of 2 tumors. Fig.2.(c) and (d) represents the levelset functions before and after segmentation. In (c) we can observe connected region as shown with red curve where as in (d) we can clearly find out the separated regions in 3-D. .

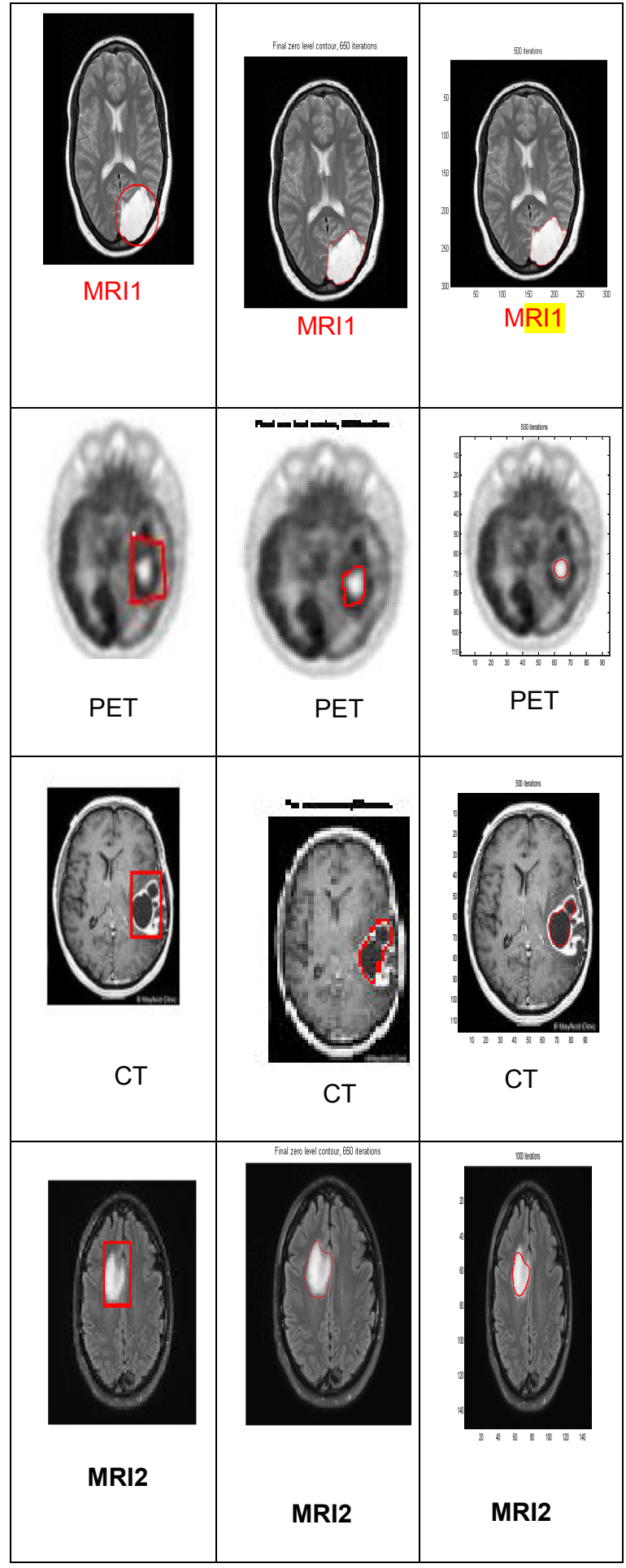

(a)

(b)

(c)

Fig 2: Segmentation of tumors from MRI,CT and PET images

(a) Original images with region of interest

(b) DRLSE segmentation

(c) Energy variational method 
Table 1. Performance Comparison

\begin{tabular}{|c|c|c|}
\hline Computation time & DRLSE & Variational \\
& Method & Method \\
\hline MRI1 & 16.484000 & 37.281000 \\
\hline PET & 10.094000 & 13.234000 \\
\hline CT & 18.344000 & 34.652000 \\
& & \\
\hline MRI2 & 15.782000 & 30.218000 \\
& & \\
\hline
\end{tabular}

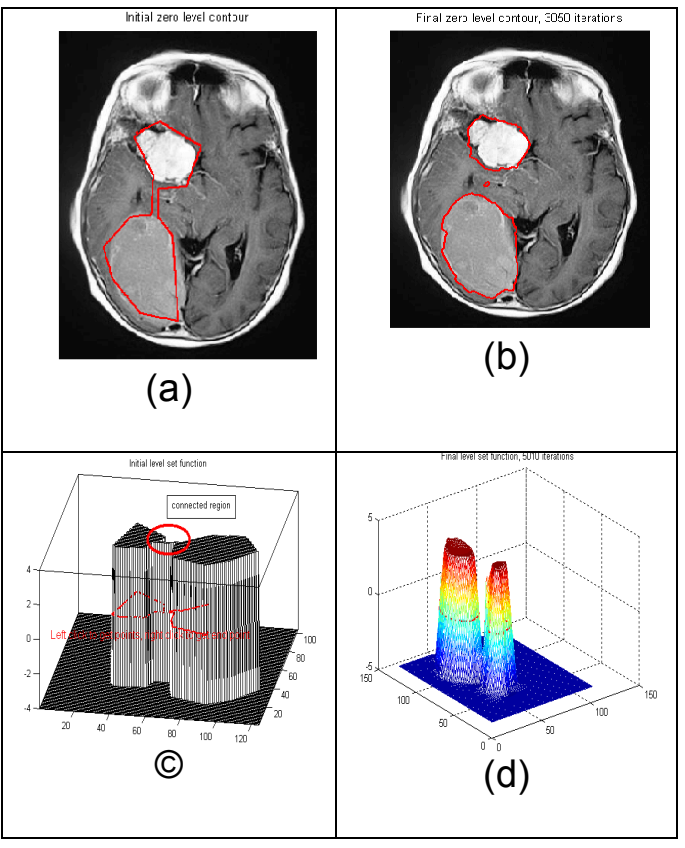

DRLSE Method

(a) initial contour (b)curve evolution

(C) initial levelset (d)final levelset

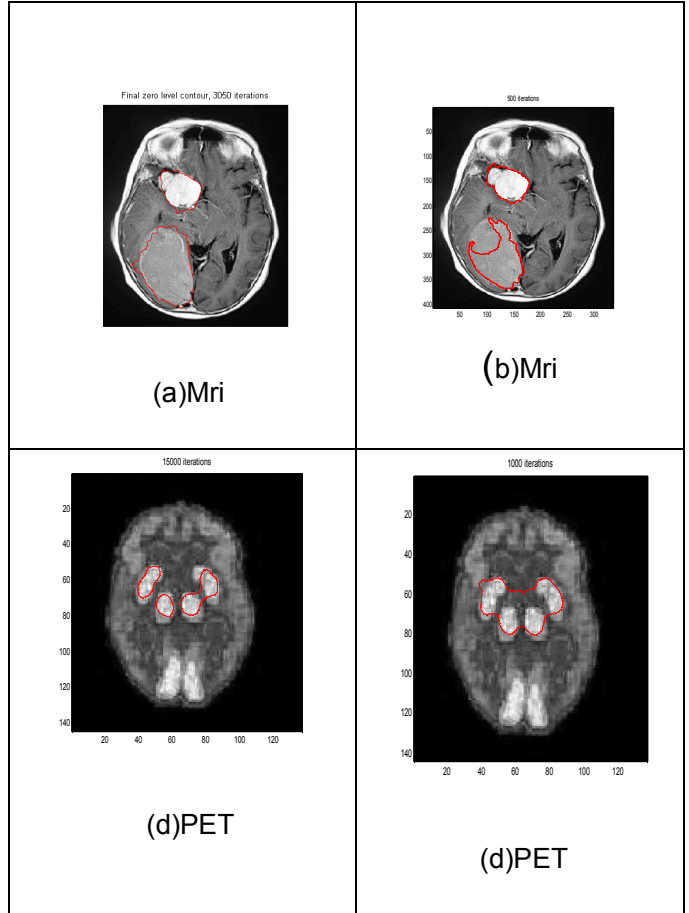

Fig 4 : DRLSE and variational methods

(a) \& (d) Mri, PET -DRLSE(proper segmentation)

(C) \& (d) Mri, PET -Variational method (improper segmentation)

Table.2. Performance Comparison

\begin{tabular}{|c|c|c|c|}
\hline & & No of iterations & $\begin{array}{c}\text { Computation } \\
\text { time(sec) }\end{array}$ \\
\hline \multirow{2}{*}{ MRI } & DRLSE & 5000 & 28.359000 \\
\hline & Variational & 1500 & \\
\hline \multirow{2}{*}{ PET } & DRLSE & 1500 & 47.953000 \\
\hline & Variational & 600 & \\
\hline
\end{tabular}


Fig.4. is the example to show the merit of DRLSE over vatiational method. Row1 is mri .Fig.4.(a) represents the proper segmentation of 2 tumors using drlse. Fig.4 (b) shows the improper segmentation. The same case we can findout with PET images in fig.4.row2,If we compare the performance quantitatively in this case we can observe that DRLSE uses more iterations and less computational time as shown in table2,columns 3,4 ,rows $2 \& 4$.

Finally it is observed that segmentation is accurate in case of DRLSE than variational .In DRLSE larger time steps can be used which reduces the computational time Though it uses more no. of iterations which is cleared from the tables 1 and 2 . In segmentation process convergence and computational speed plays the major role hence DRLSE is more suitable for medical image segmentation.

\section{CONCLUSIONS}

DRLSE formulation has an intrinsic capability of maintaining regularity of the level set function, particularly the desirable signed distance property in a vicinity of the zero level set, which ensures accurate computation and stable level set evolution. DRLSE can be implemented by a simpler and more efficient numerical scheme than conventional level set methods. DRLSE also allows more flexible and efficient initialization than generating a signed distance function as the initial LSF. This active contour model in DRLSE formulation allows the use of relatively large time steps to significantly reduce iteration numbers and computation time.

\section{REFERENCES}

[1] M. Kass, A. Witkin, and D. Terzopoulos, "Snakes: Active contour models," Int. J. Comput. Vis., vol. 1, no. 4, pp. 321-331, Jan. 1987.

[2] V. Caselles, F. Catte, T. Coll, and F. Dibos, "A geometric model for active contours in image processing", Numer. Math., vol. 66, pp. 1-31, 1993.

[3] V. Caselles, R. Kimmel, and G. Sapiro, "A geometric model for active contours”, IJCV, vol. 22, pp. 61-79, 1997.
[4] Mark Holden, "A Review of Nonrigid Transformations for Medical Images" Ieee Transactions On Medical Imaging, Vol. 27, No. 1, January 2008.

[5] J.A. Sethian, "Level Set Methods and Fast Marching Methods", Cambridge University, 1999.

[6] S. Osher, R. Fedkiw, "Level Set Methods and Dynamic Implicit Surface”, Springer-Verlag, 2002.

[7] D. Adalsteinsson, J.A. Sethian, "A Fast Level Set Method for Propagating Interfaces”, J.Comp.Phys., pp.269 277, 1995.

[8] J. V. Hajnal, D. L. G. Hill, and D. J. Hawkes, Eds., Medical ImageRegistration. Bocal Raton, FL: CRC Press, 2001.

[9] J. Maintz and M. A. Viergever, "A survey of medical image registration,"Med. Imag. Anal., vol. 2, no. 1, pp. 136, 1998.

[10] R.Malladi, J.Sethian, and B.Vemuri. Shape modeling with front propagation:A level set approach. IEEE Trans. on Pattern Analysis and Machine Intelligence, V.17, No.2, pp.158-175, February 1995.

[11] T.Chan, L.A. Vese, "Active Contours without Edges", IEEE Transaction on Image Processing, vol.10(2), pp.266 277, 2001.

[12] Chunming Li 1, Chenyang Xu 2, Changfeng Gui 3, and Martin D. Fox, "Level Set Evolution without Reinitialization: A New Variational Formulation", 2005 IEEE Computer Society Conference on Computer Vision and Pattern Recognition, 2005.

[13] Chunming et.al. "A Variational Level Set Approach to Segmentation and Bias Correction of Images with Intensity Inhomogeneity”,MICCAI 2008, Part II, LNCS 5242, pp. 1083-1091, 2008.

[14] Chunming Li, Chenyang Xu, Senior Member, IEEE, Changfeng Gui, and Martin D. Fox, Member, IEEE," Distance Regularized Level Set Evolution and Its Application to Image Segmentation", IEEE Transactions On Image Processing, Vol. 19, No. 12, December 2010, pp3243-3254. 